\title{
Molecular Mechanism of Thiazolidinedione-Mediated Inhibitory Effects on Osteoclastogenesis
}

\author{
Dongfeng Zhao ${ }^{1,2}$, Zhenqi Shi ${ }^{2}$, Amy H. Warriner ${ }^{3}$, Ping Qiao ${ }^{2}$, Huixian Hong ${ }^{2}$, Yongjun Wang ${ }^{1 *}$, \\ Xu Feng ${ }^{2 *}$
}

1 Longhua Hospital, Shanghai University of Traditional Chinese Medicine, Shanghai, People's Republic of China, 2 Department of Pathology, University of Alabama at Birmingham, Birmingham, Alabama, United States of America, 3 Department of Medicine, University of Alabama at Birmingham, Birmingham, Alabama, United States of America

\begin{abstract}
Thiazolidinediones are synthetic peroxisome proliferator-activated receptor $\gamma$ agonists used to treat type 2 diabetes mellitus. Clinical evidence indicates that thiazolidinediones increase fracture risks in type 2 diabetes mellitus patients, but the mechanism by which thiazolidinediones augment fracture risks is not fully understood. Several groups recently demonstrated that thiazolidinediones stimulate osteoclast formation, thus proposing that thiazolidinediones induce bone loss in part by prompting osteoclastogenesis. However, numerous other studies showed that thiazolidinediones inhibit osteoclast formation. Moreover, the molecular mechanism by which thiazolidinediones modulate osteoclastogenesis is not fully understood. Here we independently address the role of thiazolidinediones in osteoclastogenesis in vitro and furthermore investigate the molecular mechanism underlying the in vitro effects of thiazolidinediones on osteoclastogenesis. Our in vitro data indicate that thiazolidinediones dose-dependently inhibit osteoclastogenesis from bone marrow macrophages, but the inhibitory effect is considerably reduced when bone marrow macrophages are pretreated with RANKL. In vitro mechanistic studies reveal that thiazolidinediones inhibit osteoclastogenesis not by impairing RANKLinduced activation of the NF- $\mathrm{KB}, \mathrm{JNK}, \mathrm{p} 38$ and ERK pathways in bone marrow macrophages. Nonetheless, thiazolidinediones inhibit osteoclastogenesis by suppressing RANKL-induced expression of NFATc1 and c-Fos, two key transcriptional regulators of osteoclastogenesis, in bone marrow macrophages. In addition, thiazolidinediones inhibit the RANKL-induced expression of osteoclast genes encoding matrix metalloproteinase 9, cathepsin K, tartrate-resistant acid phosphatase and carbonic anhydrase II in bone marrow macrophages. However, the ability of thiazolidinediones to inhibit the expression of NFATc1, c-Fos and the four osteoclast genes is notably weakened in RANKL-pretreated bone marrow macrophages. These in vitro studies have not only independently demonstrated that thiazolidinediones exert inhibitory effects on osteoclastogenesis but have also revealed crucial new insights into the molecular mechanism by which thiazolidinediones inhibit osteoclastogenesis.
\end{abstract}

Citation: Zhao D, Shi Z, Warriner AH, Qiao P, Hong H, et al. (2014) Molecular Mechanism of Thiazolidinedione-Mediated Inhibitory Effects on Osteoclastogenesis. PLoS ONE 9(7): e102706. doi:10.1371/journal.pone.0102706

Editor: Jean-Marc Vanacker, Institut de Génomique Fonctionnelle de Lyon, France

Received March 7, 2014; Accepted June 23, 2014; Published July 17, 2014

Copyright: (c) 2014 Zhao et al. This is an open-access article distributed under the terms of the Creative Commons Attribution License, which permits unrestricted use, distribution, and reproduction in any medium, provided the original author and source are credited.

Data Availability: The authors confirm that all data underlying the findings are fully available without restriction. Data are included within the paper.

Funding: Research reported in this publication was supported by the National Institute of Arthritis and Musculoskeletal and Skin Diseases, part of the National Institutes of Health, under Award Number AR47830. This work was also supported by grants from the National Basic Research Program of China (2010CB530400) the Key Program of Natural Science Foundation of China (30930111), and the Program for Changjiang Scholars and Innovative Research Team in University (PCSIRT, IRT1270). The funders had no role in study design, data collection and analysis, decision to publish, or preparation of the manuscript.

Competing Interests: The authors have declared that no competing interests exist.

*Email: yjwang88@hotmail.com (YW); xufeng@uab.edu (XF)

\section{Introduction}

Types 2 diabetes mellitus (T2DM) accounts for 90-95\% of all diabetes cases and causes significant economic and societal burdens globally $[1,2]$. Thiazolidinediones (TZDs) represent one class of drugs used to treat $\mathrm{T} 2 \mathrm{DM}$ and they promote insulin sensitivity to enhance glucose consumption [3,4]. TZDs are highly effective oral mediators that exert their anti-hyperglycemic function through targeting peroxisome proliferator-activated receptor $\gamma(\operatorname{PPAR} \gamma)[5,6]$, a master regulator of glucose metabolism $[7,8]$. In addition, PPAR $\gamma$ also regulates various other biological processes including adipogenesis, lipid metabolism, macrophage biology and inflammation [9-14].

However, clinical evidence has established that TZD treatments increase the risk of fracture in patients with T2DM [15-17].
Available data indicate that TZDs modulate bone remodeling through various bone cells including osteoblasts, osteocytes and osteoclasts [18]. It has been shown that TZDs induce preferential differentiation of mesenchymal stem cells into adipocytes instead of osteoblasts by activating PPAR $\gamma$ [19], leading to bone loss and structural deterioration [20,21]. Moreover, TZDs also negatively affect bone formation by inducing the apoptosis of osteocytes and increasing expression of sclerostin $[22,23]$. On the other hand, while a number of groups have also investigated the effects of TZDs on osteoclastogenesis [24-32], these studies generated controversial findings. Among these studies, seven demonstrated that TZDs exert inhibitory effects on osteoclastogenesis [24-30]; in contrast, two investigations showed that these PPAR $\gamma$ agonists promote osteoclast formation in an PPAR $\gamma$-dependent manner $[31,32]$. 
Osteoclasts, the multinucleated bone-resorbing cells, not only play a crucial role in skeletal development and adult bone remodeling but are also implicated in the pathogenesis of various bone disorders $[33,34]$. Osteoclasts differentiate from mononuclear cells of the monocyte/macrophage lineage upon stimulation by the monocyte/macrophage colony stimulating factors (M-CSF) and the receptor activator of $\mathrm{NF}-\kappa \mathrm{B}$ ligand (RANKL) [35]. Whereas M-CSF mainly stimulates proliferation and survival of bone marrow macrophages (BMMs, namely, osteoclast precursors), RANKL primarily drives osteoclast differentiation. RANKL binds to its receptor RANK, a member of the TNF receptor superfamily, to activate numerous signaling pathways $(\mathrm{NF}-\kappa \mathrm{B}$, JNK, ERK, p38 and Akt) [35,36]. RANKL also up-regulates the expression of nuclear factor of activated T-cells cl (NFATc1), which plays an essential role in osteoclastogenesis [37]. c-Fos is another factor crucially involved in osteoclastogenesis [38]. RANKL increases the expression of c-Fos which cooperates with NFATcl to regulate gene expression during osteoclastogenesis [37]. Thus, these RANKL-activated signaling pathways promote osteoclastogenesis in part by regulating the expression of genes including those encoding matrix metalloproteinase 9 (MMP9), cathepsin $\mathrm{K}$ (Ctsk), tartrate-resistant acid phosphatase (TRAP) and carbonic anhydrase II (Car2) [39,40].

RANKL promotes osteoclastogenesis in part by committing BMMs into the osteoclast lineage [41]. Moreover, we and others have previously revealed that RANKL-mediated osteoclast lineage commitment of BMMs determines the effects of numerous factors on osteoclastogenesis. Lipopolysaccharide (LPS), a common bacteria-derived product implicated in the periodontal bone loss, inhibits osteoclastogenesis from freshly isolated BMMs but stimulates osteoclast formation from RANKL-pretreated BMMs $[42,43]$. Furthermore, whereas interferon- $\gamma($ IFN- $\gamma)$, a potent antiosteoclastogenic factor, strongly inhibits osteoclastogenesis from fresh BMMs, the suppressive effect is significantly reduced when osteoclast precursors are pre-exposed to RANKL [44,45]. IL-1 and TNF- $\alpha$, two proinflammatory cytokines, are unable to promote osteoclastogenesis without RANKL. However, they can do so in the presence of low levels of RANKL or with RANKL pretreatment [46-49].

In this work, given the controversy on the role of TZDs in osteoclastogenesis, first we independently and thoroughly carried out a series of in vitro assays to further investigate the effects of TZDs on osteoclastogenesis. In particular, we performed the assays with rosiglitazone and pioglitazone, which are two common forms of TZDs used to treat T2DM, in parallel to obtain more convincing data. Moreover, given that RANKL-mediated osteoclast lineage commitment of BMMs has been shown to determine the effects of LPS, IFN- $\gamma$, IL- 1 and TNF- $\alpha$ on osteoclastogenesis, we also examined whether RANKL-mediated osteoclast lineage commitment alters the effect of TZDs on osteoclastogenesis. Finally, we carried out mechanistic studies to gain insights into the molecular mechanism by which TZDs modulate osteoclastogenesis.

\section{Materials and Methods}

\section{Chemicals, biological reagents and mice}

Rosiglitazone (R2408), pioglitazone hydrochloride (E6910) and dimethyl sulfoxide (DMSO) (D2438) were purchased from SigmaAldrich (St. Louis, MO). Stock solutions $\left(2 \times 10^{4} \mu \mathrm{M}\right)$ of rosiglitazone and pioglitazone were prepared in DMSO monthly and stored at $4^{\circ} \mathrm{C}$. Recombinant purified glutathione S-transferaseRANKL was purified as described previously [50]. Mouse M-CSF was prepared from an M-CSF-producing cell line as previously described [51]. Recombinant mouse TNF- $\alpha$ (410-TRNC-050) was purchased from R\&D system (Minneapolis, MN). The following antibodies were purchased from Cell Signaling Technology, Inc.

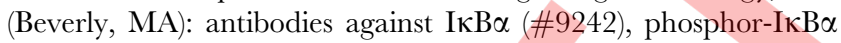
(\#2859s), p44/42ERK (\#9102), phospho-44/42ERK (\#9101s), JNK (\#9252), phospho-JNK (\#9251s), p38 (\#9212), phosphop38 (\#9211s), and lamin A/C (\#2032s). Antibodies against NFATcl (sc-7294), RANK (sc-7624) and c-Fos (sc-253) were purchased from Santa Cruz Biotechnology (Santa Cruz, CA). Catalog numbers for the chemicals and biological reagents are provided in parentheses. C57BL/6 mice were purchased from Harlan Industries (Indianapolis, IN), and were maintained in the animal facility at University of Alabama at Birmingham (UAB). The study was approved by UAB institutional animal care and use committee (IACUC) (animal protocol number: 130407854). The experiments involving mice were performed in accordance with the Instructions and Regulations of UAB IACUC.

\section{In vitro osteoclastogenesis assays}

BMMs were isolated from long bones of 4-to-6-week-old male and female C57BL/ 6 mice. To generate osteoclasts, $5 \times 10^{4}$ cells were plated per well in 24-well tissue culture plates and treated with different doses of M-CSF and RANKL as indicated in individual experiments. Osteoclasts were stained for TRAP activity with a leukocyte acid phosphatase commercial kit (Sigma, 387-A). The images of osteoclastogenesis assays were obtained using Olympus IX70 Fluorescence Microscope (Shinjuku, Japan).

\section{In vitro bone resorption assays}

BMMs, $5 \times 10^{4}$ cells per/well, were seeded on bovine cortical bone slices in 24-well tissue culture plates. Cells were treated under conditions indicated in individual experiments to promote osteoclastogenesis and bone resorption for up to 10 days. Then, bone slices were harvested, and cells were removed from bone slices with $0.25 \mathrm{M}$ ammonium hydroxide and mechanical agitation. Bone slices were subjected to scanning electron microscopy (SEM) using a Philips 515 SEM (Materials Engineering Department, UAB). Bone resorption surfaces were quantified by measuring the percentage of the pits area in 5 random areas using the ImageJ analysis software obtained from National Institutes of Health.

\section{Western blot assays}

For the assays performed to assess the activation of I $\mathrm{B} \alpha / \mathrm{NF}$ $\kappa \mathrm{B}, \mathrm{ERK}, \mathrm{JNK}$, p38 pathways, BMMs were washed twice with ice cold PBS and then lysed with the lysis buffer from Cell Signaling Technology (\#9803s), supplemented with protease inhibitor (\#8340) and phosphatase inhibitor cocktail 1 (\#2850) and 2 (\#5726) from Sigma-Aldrich. For NFATcl, RANK and c-Fos expression assays, BMMs were washed twice with ice cold PBS and then lysed with the lysis buffer from Cell Signaling Technology (\#9803), supplemented with protease inhibitor (\#8340). Cytoplasmic and nuclear extracts were prepared using the NE-PER Nuclear and Cytoplasmic Extraction Reagents (Catalog No: 78833) from Thermo Scientific (Rockford, IL). Lysates were then subjected to Western blot analysis as described in our previous study [47] except that the primary antibodies against NFATc1, RANK and c-Fos were incubated in 5\% nonfat dry milk solution (TBS containing $0.1 \%$ Tween 20). Membranes were washed extensively, and an ECL detection assay was performed using a Super Signal West Dura kit from Pierce. Densitometric analysis of Western blot bands was performed using FluiorChem 8900 imager from Alpha Innotech (Santa Clara, CA). 
A M-CSF
RANKL
Ros
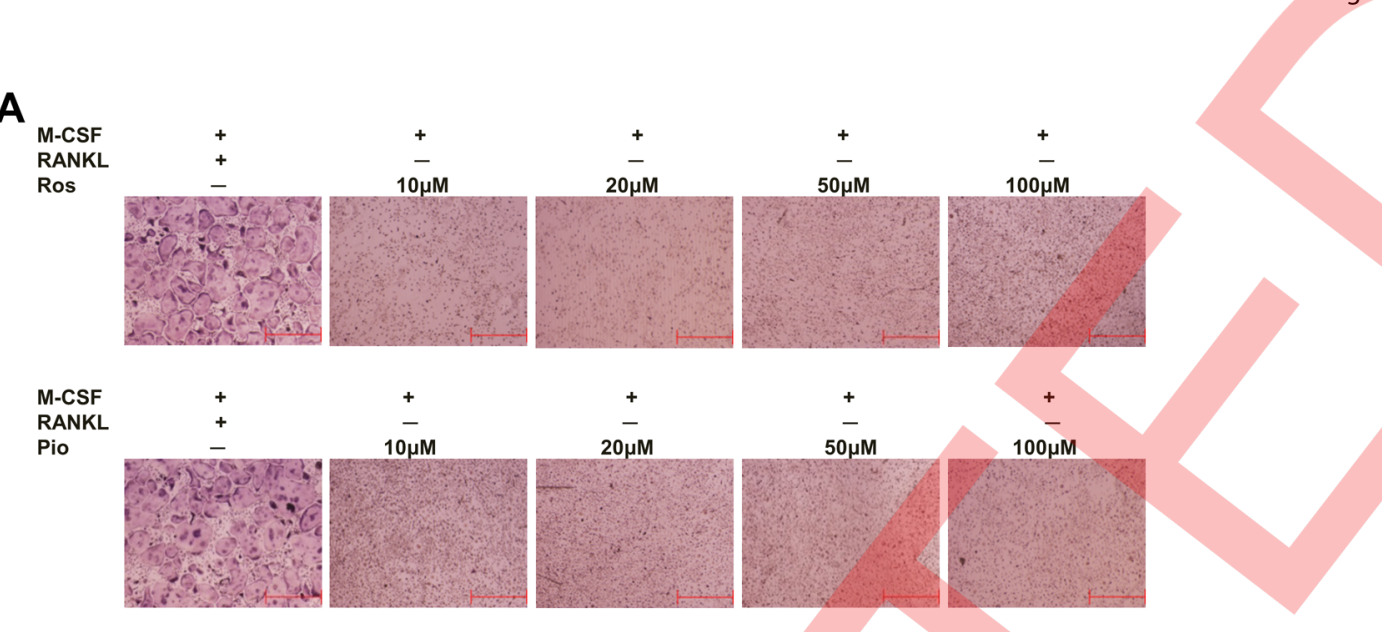

B
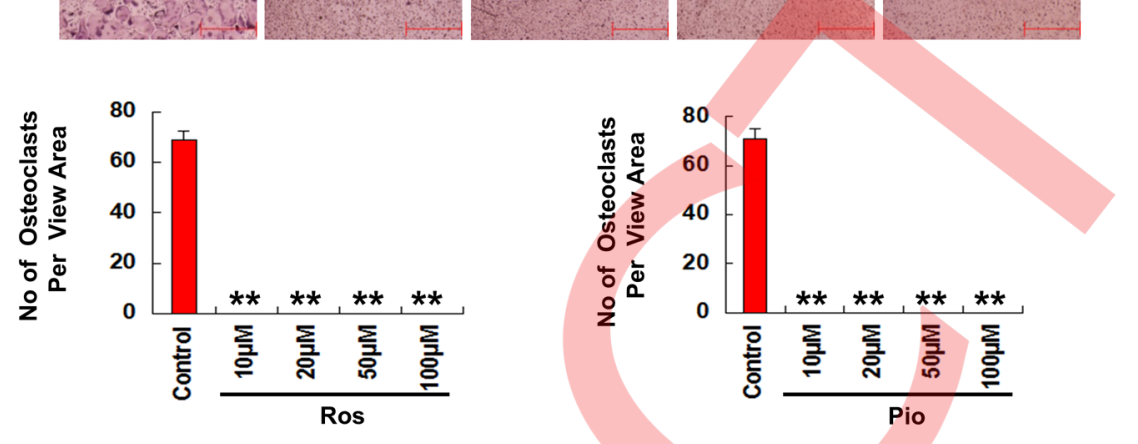

Figure 1. Rosiglitazone and pioglitazone do not induce osteoclastogenesis in the absence of RANKL. (A) BMMs were treated with M-CSF $(44 \mathrm{ng} / \mathrm{ml})$ and RANKL $(100 \mathrm{ng} / \mathrm{ml})$ as control, or M-CSF $(44 \mathrm{ng} / \mathrm{ml})$ plus different doses $(10 \mu \mathrm{M}, 20 \mu \mathrm{M}, 50 \mu \mathrm{M}$ and $100 \mu \mathrm{M})$ of rosiglitazone (Ros) or pioglitazone (Pio) for 4 days. The cultures were stained for TRAP activity. All assays were performed in triplicate and repeated 3 times. One representative view from each condition is shown. (B) Quantification of the osteoclastogenesis assays in A for Ros (left panel) and Pio (right panel). The number of multinucleated TRAP-positive cells ( $>3$ nuclei) per representative view area ( $40 \times$ magnification) was obtained. Bars show averages of three replicates \pm S.D. TZD-treated cultures were compared with control cultures. ${ }^{* *}$ : $\mathrm{P}<0.001$.

doi:10.1371/journal.pone.0102706.g001

\section{Semi-quantitative RT-PCR analysis}

Total RNA was isolated from BMMs using TRIzol reagent from Invitrogen (Carlsbad, CA). Reverse transcription was performed using the Superscript III RT-PCR system (Invitrogen) according to standard protocol. In brief, $2 \mu \mathrm{g}$ of total RNA was reverse-transcribed to cDNA with oligo $(\mathrm{dT})$ in a $20 \mu \mathrm{l}$ volume at $50^{\circ} \mathrm{C}$ for $60 \mathrm{~min}$. The reverse transcription reaction was then followed by enzyme inactivation and RNA $\mathrm{H}$ digestion. PCR amplification of the MMP9, Ctsk, TRAP, Car2, and GAPDH genes was carried out as in our previous [47]. $25 \mu \mathrm{l}$ of PCR products was loaded and separated on $2 \%$ agarose gel for electrophoretic analysis. The images of PCR product bands were obtained using FluorChem 8900 imager from Alpha Innotech Corporation (San Leandro, CA).

\section{Statistical analysis}

Bone resorption data are expressed as mean \pm S.D. of the percentage of resorbed bone surface and osteoclastogenesis assay data are expressed as mean \pm S.D. of the number of TRAPpositive cells/view area at $40 \times$ magnification. Statistical analyses were carried out using ANOVA with Bonferroni adjustment or Student's $t$-test. $p$ values less than 0.05 are considered significant.

\section{Results}

\section{TZDs dose-dependently inhibit osteoclastogenesis}

Given the controversy on the effect of TZDs on osteoclastogenesis, in this study we seek to perform a series of experiments to fully and independently investigate the role of TZDs in osteoclast biology. It has been established that M-CSF and RANKL are two essential and sufficient factors for osteoclastogenesis [35]. While M-CSF functions to stimulate proliferation and survival of osteoclast precursors, RANKL is the key factor driving osteoclast differentiation. Therefore, our initial set of experiments aims to address whether TZDs can replace RANKL in promoting osteoclastogenesis in the presence of M-CSF. BMMs were treated with M-CSF and RANKL as positive control, or with M-CSF plus different doses of rosiglitazone or pioglitazone in tissue culture plates to promote osteoclastogenesis (Figure 1). While RANKL treatment led to the formation of osteoclasts, rosiglitazone and pioglitazone failed to induce osteoclastogenesis, indicating that TZDs cannot replace RANKL in promoting osteoclastogenesis.

Previously, we showed that whereas TNF- $\alpha$ or IL- 1 cannot stimulate osteoclastogenesis in the presence of M-CSF, they can do so with low/permissive levels (10 ng.ml) of RANKL or RANKL pretreatment $[47,52]$. Therefore, we performed assays to determine whether TZDs can induce osteoclastogenesis with low levels of RANKL or RANKL pretreatment. As shown in Figure 2A-2B, $\mathrm{TNF}-\alpha(5 \mathrm{ng} / \mathrm{ml})$ induced the formation of numerous osteoclasts in the presence of $10 \mathrm{ng} / \mathrm{ml}$ RANKL. However, rosiglitazone and pioglitazone at concentrations as high as $100 \mu \mathrm{M}$ were unable to promote osteoclastogenesis in the presence of $10 \mathrm{ng} / \mathrm{ml}$ RANKL. Similarly, TNF- $\alpha(5 \mathrm{ng} / \mathrm{ml})$ promoted osteoclastogenesis from BMMs pretreated with $100 \mathrm{ng} / \mathrm{ml}$ RANKL for 24 hours (Figure 2C-2D), rosiglitazone and pioglitazone at concentrations as high as $100 \mu \mathrm{M}$ failed to do so from RANKL-pretreated BMMs. These data indicate that TZDs are unable to promote osteoclastogenesis with low levels of RANKL or RANKL pretreatment. 
A

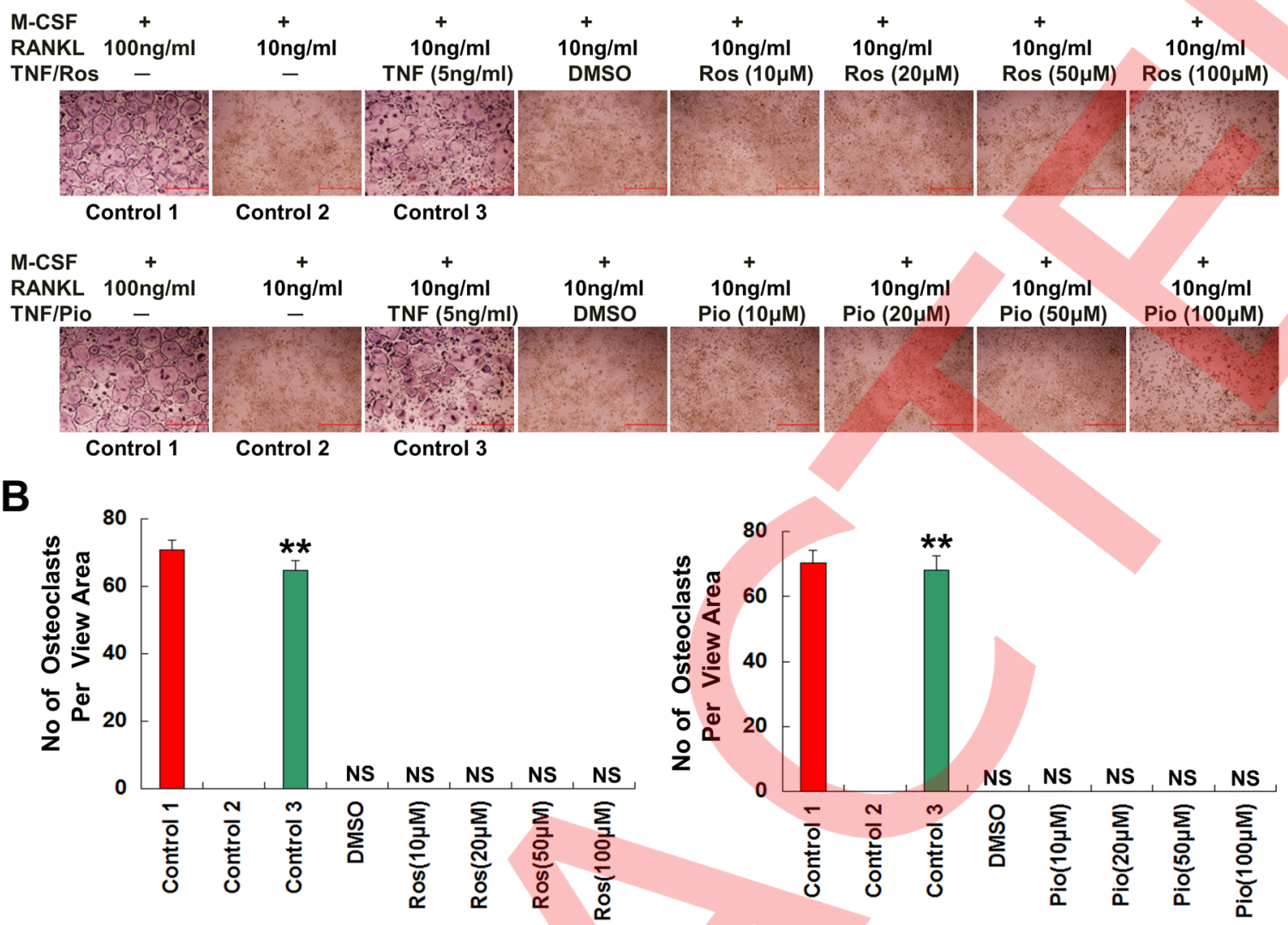

C
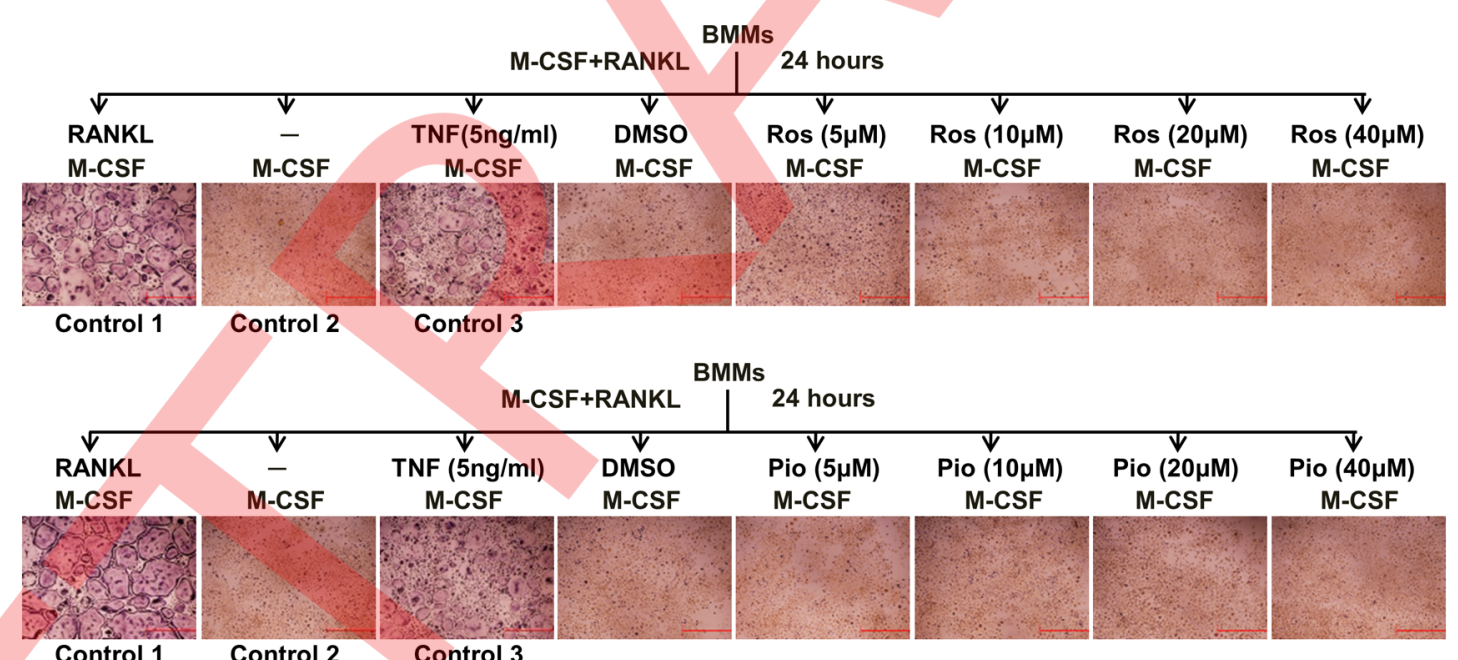

\section{D}
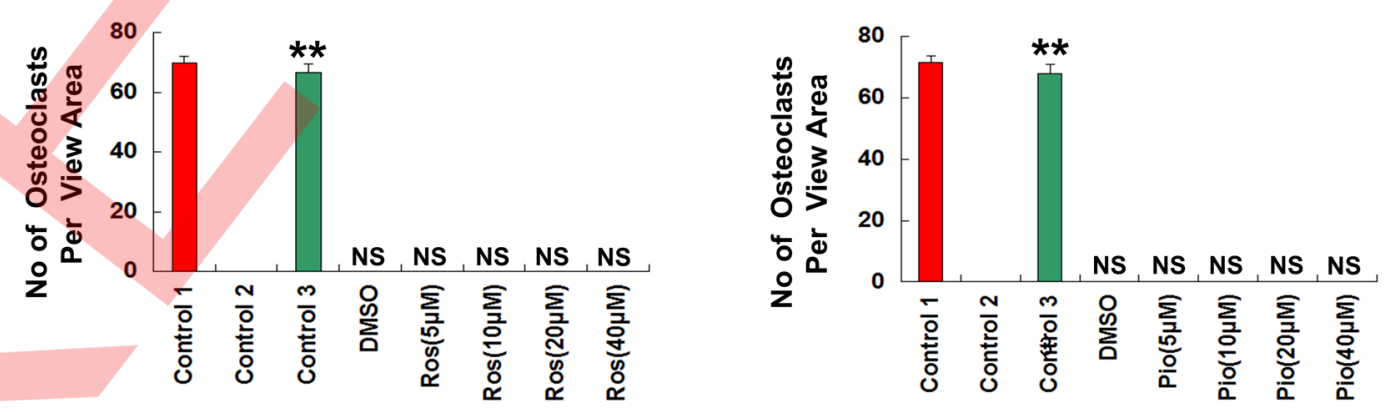
Figure 2. Rosiglitazone and pioglitazone do not induce osteoclastogenesis with low levels of RANKL or RANKL pretreatment. (A) BMMs were treated with M-CSF (44 $\mathrm{ng} / \mathrm{ml})$ and RANKL $(100 \mathrm{ng} / \mathrm{ml})$ as control 1, M-CSF $(44 \mathrm{ng} / \mathrm{ml})$ and RANKL $(10 \mathrm{ng} / \mathrm{ml})$ as control 2, M-CSF (44 $\mathrm{ng} /$ $\mathrm{ml})$ and RANKL $(10 \mathrm{ng} / \mathrm{ml})$ plus TNF- $\alpha(\mathrm{TNF}, 5 \mathrm{ng} / \mathrm{ml})$ as control 3, M-CSF $(44 \mathrm{ng} / \mathrm{ml})$ and RANKL $(10 \mathrm{ng} / \mathrm{ml})$ plus DMSO or different doses $(5 \mu \mathrm{M}$, $10 \mu \mathrm{M}, 20 \mu \mathrm{M}$ or $40 \mu \mathrm{M}$ ) of rosiglitazone (Ros) or pioglitazone (Pio) for 4 days and then stained for TRAP activity. The assays were performed in triplicate and repeated 3 times. One representative view from each condition is shown. (B) Quantification of the osteoclastogenesis assays in A for Ros (left panel) and Pio (right panel). The number of multinucleated TRAP-positive cells ( $>3$ nuclei) per representative view area (40 $\times$ magnification) was obtained. Bars show averages of three replicates \pm S.D. Control 3, DMSO, or TZD-treated cultures were compared with control 2. ${ }^{* *}$ : $\mathrm{P}<0.001$. NS: not significant. (C) BMMs were treated with M-CSF $(44 \mathrm{ng} / \mathrm{ml})$ and RANKL $(100 \mathrm{ng} / \mathrm{ml})$ for 24 hours and then were continued with M-CSF (44 $\mathrm{ng} / \mathrm{ml})$ and RANKL $(100 \mathrm{ng} / \mathrm{ml})$ as control 1, M-CSF (44 ng/ml) alone as control 2, M-CSF $(44 \mathrm{ng} / \mathrm{ml})$ and TNF $(5 \mathrm{ng} / \mathrm{ml})$ as control 3, M-CSF $(44 \mathrm{ng} / \mathrm{ml})$ and DMSO, or M-CSF (44 ng/ml) plus different doses $(5 \mu \mathrm{M}, 10 \mu \mathrm{M}, 20 \mu \mathrm{M}$ or $40 \mu \mathrm{M})$ of Ros or Pio for additional 3 days. The cultures were stained for TRAP activity. The experiments were performed in triplicate and repeated 3 times. One representative view from each condition is shown. (D) Quantification of the osteoclastogenesis assays in A for Ros (left panel) and Pio (right panel). The number of multinucleated TRAP-positive cells ( $>$ 3 nuclei) per representative view area (40 ×magnification) was obtained. Bars show averages of three replicates \pm S.D. Control 3, DMSO, or TZD-treated cultures were compared with control 2. **: P<0.001. NS: not significant. doi:10.1371/journal.pone.0102706.g002

Having shown that TZDs are incapable of inducing osteoclastogenesis in the absence of RANKL, in the presence of low levels of RANKL, or with RANKL pretreatment, we further investigated their effects on osteoclastogenesis with optimal levels (100 ng/ $\mathrm{ml}$ ) of RANKL. We found that both rosiglitazone and pioglitazone dose-dependently inhibited RANKL-mediated osteoclastogenesis with complete inhibition of osteoclastogenesis at $40 \mu \mathrm{M}$ (Figure 3). Bone surfaces represent the physiological substratum for osteoclastogenesis. To further investigate the effect of TZDs on osteoclastogenesis in a more physiologically relevant manner, we repeated the assays on bone slices. The data reveal that both rosiglitazone and pioglitazone treatment led to a significant reduction in bone resorption at $20 \mu \mathrm{M}$ and complete absence of bone resorption at $40 \mu \mathrm{M}$ (Figure 4), further indicating that TZDs inhibit RANKL-induced osteoclastogenesis.

TZDs do not affect RANKL-induced activation of the NF$\kappa B$ and MAPK pathways in BMMs

To elucidate the molecular mechanism by which TZDs inhibit osteoclastogenesis, we first examine whether TZDs exert an effect

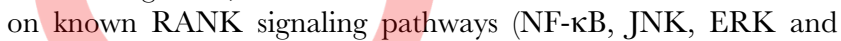
p38) involved in osteoclastogenesis. BMMs were treated with $\mathrm{M}$ CSF and RANKL only, or M-CSF and RANKL plus vehicle

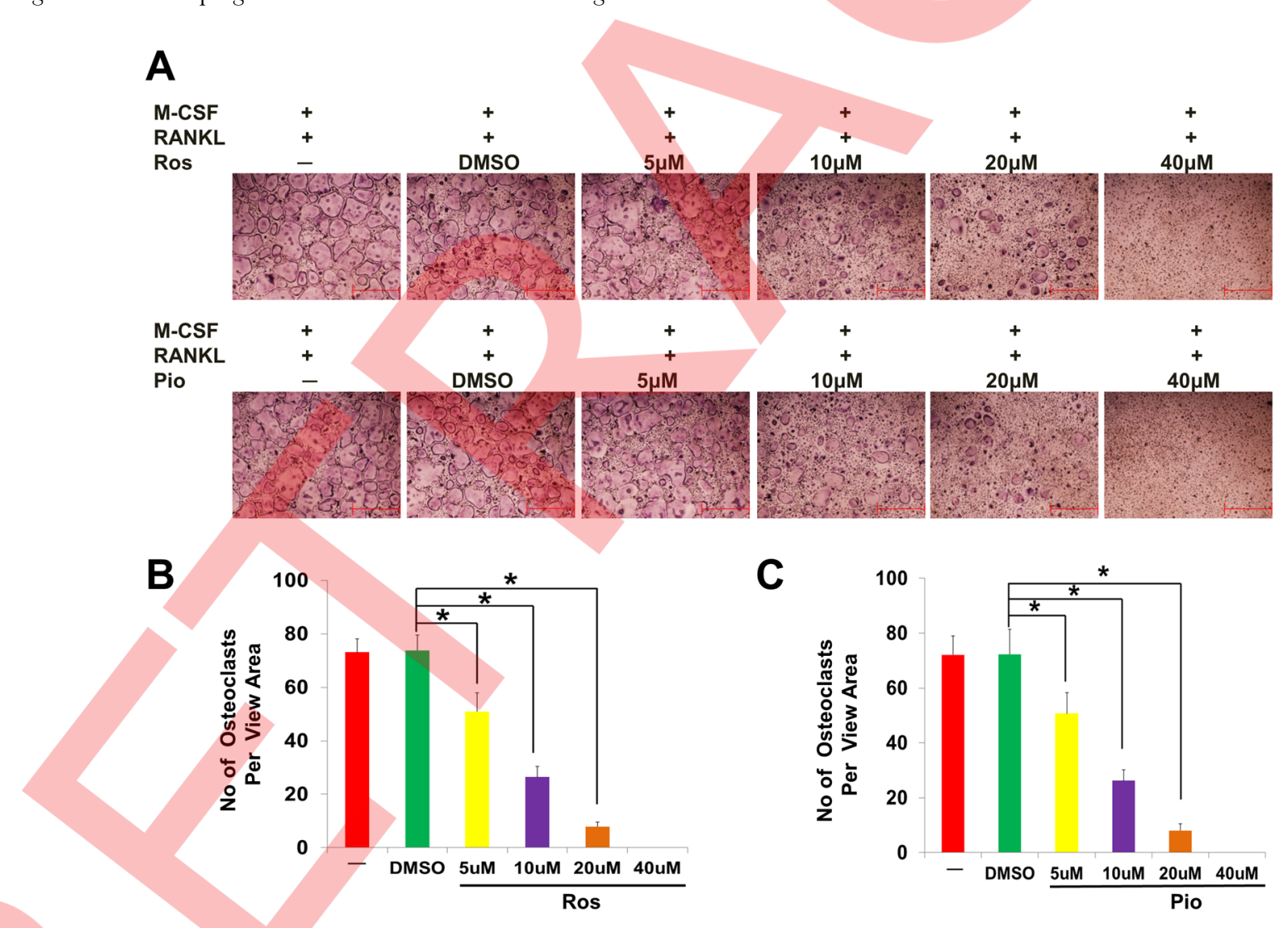

Figure 3. Rosiglitazone and pioglitazone inhibit RANKL-mediated osteoclastogenesis in tissue culture plates. (A) BMMs were treated with M-CSF $(44 \mathrm{ng} / \mathrm{ml})$ and RANKL $(100 \mathrm{ng} / \mathrm{ml})$, or M-CSF $(44 \mathrm{ng} / \mathrm{ml})$ and RANKL $(100 \mathrm{ng} / \mathrm{ml})$ plus vehicle (DMSO) or different doses $(5 \mu M, 10 \mu M$, $20 \mu \mathrm{M}$ or $40 \mu \mathrm{M}$ ) of rosiglitazone (Ros) or pioglitazone (Pio) for 4 days. The cultures were then stained for TRAP activity. All assays were performed in triplicate and repeated 3 times. One representative view from each condition is shown. (B) Quantification of osteoclastogenesis assays for Ros in A. (C) Quantification of osteoclastogenesis assays for Pio in A. The number of multinucleated TRAP-positive cells ( $>3$ nuclei) per representative view area at $40 \times$ magnification was obtained. Bars show averages of three replicates \pm S.D. ${ }^{*} P<0.05$. doi:10.1371/journal.pone.0102706.g003 
A
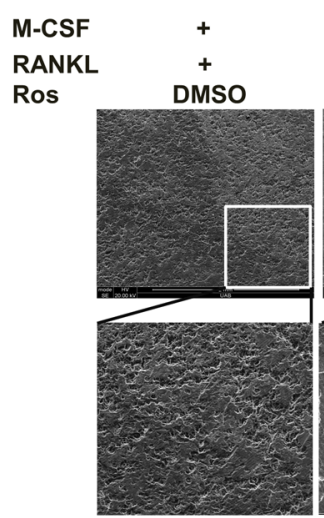

C

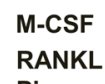

Pio
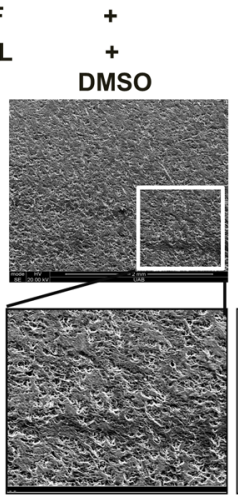

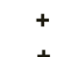

$+$ $20 \mu M$
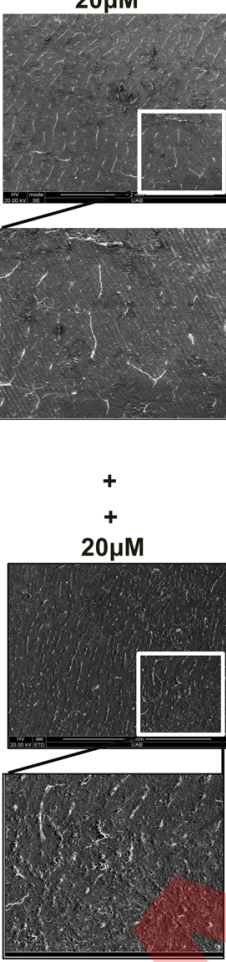

西

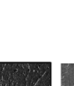

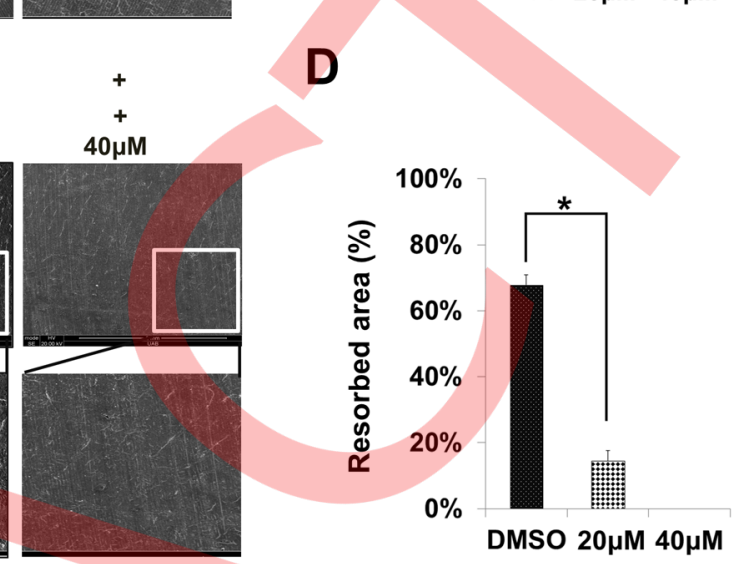

B

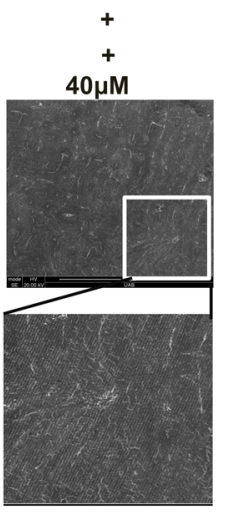

Figure 4. Rosiglitazone and pioglitazone significantly inhibit the formation of functional osteoclasts on bone slices. (A) BMMs were seeded on bone slices and treated with M-CSF ( $44 \mathrm{ng} / \mathrm{ml}$ ) and RANKL (100 ng/ml) plus vehicle (DMSO), $20 \mu \mathrm{M}$ or $40 \mu \mathrm{M}$ of rosiglitazone (Ros) for 10 days. Bone resorption pits were visualized by SEM. One representative view from each condition is shown. (B) Quantification of resorbed bone area for Ros in A. Bars show percentage averages of resorbed bone area \pm S.D. *P $<0.05$. (C) The same assays in A were carried out with pioglitazone (Pio). (D) Quantification of resorbed bone area for Pio in C. Bars show percentage averages of resorbed bone area \pm S.D. ${ }^{*} \mathrm{P}<0.05$.

doi:10.1371/journal.pone.0102706.g004

(DMSO), rosiglitazone or pioglitazone for 5 or 10 minutes. Activation of $\mathrm{NF}-\kappa \mathrm{B}, \mathrm{JNK}$, ERK and p38 pathways was determined using Western bot analysis as the levels of phosphorylated form of IKB, JNK, ERK and p38, respectively (Figure 5). RANKL treatment led to increased phosphorylation of IкB, JNK, ERK and p38 at 5 minute (lane 2) and 10 minutes (lane 6) compared to those without RANKL treatment (lane 1), replicating previous findings that RANKL activates NF- $\mathrm{B}, \mathrm{JNK}$, ERK and p38 pathways in osteoclast precursors. However, phosphorylation of IкB, JNK, ERK and p38 were not considerably affected by DMSO (lanes 3 and 7), rosiglitazone (lanes 4 and 8 ) or pioglitazone (lanes 5 and 9). These findings indicate that rosiglitazone and pioglitazone do not exert considerable effects on the activation of the NF- $\kappa \mathrm{B}, \mathrm{JNK}, \mathrm{ERK}$ or p38 pathways in osteoclast precursors.

\section{TZDs repress RANKL-induced expression of NFATc1, c-}

Fos and osteoclast genes

NFATcl and c-Fos are two important transcriptional regulators of osteoclastogenesis $[37,38]$. To address whether TZDs exert an effect on NFATcl and c-Fos expression, BMMs were treated with $40 \mu \mathrm{M}$ rosiglitazone or pioglitazone for 1 or 2 days for Western blot analysis of NFATc1 and c-Fos expression. We found that both rosiglitazone and pioglitazone significantly suppressed RANKLinduced expression of NFATcl (lane 2 vs. lanes 3 and 4; also lane 6 vs. lanes 7 and 8, top panel, Figure 6A) and c-Fos (lane 2 vs. lanes 3 and 4; also lane 6 vs. lanes 7 and 8, bottom panel, Figure 6A) in BMMs. Notably, RANKL not only induces NFATcl expression but also activates NFATcl by promoting its nuclear translocation in osteoclast precursors [37]. Thus, we further examined whether TZDs affect NFATcl nuclear translocation in osteoclast precursors. BMMs were treated with $40 \mu \mathrm{M}$ rosiglitazone or pioglitazone for 2 days and then cytoplasmic and nuclear extracts were prepared for Western blot analysis of NFATcl levels in these subcellular fractions. We found that TZDs do not exert considerable effects on RANKL-induced nuclear translocation of NFATcl (Figure 6B). These results indicate that TZDs inhibit osteoclastogenesis in part by suppressing RANKL-induced expression of the two key transcriptional regulators of osteoclastogenesis, NFATcl and c-Fos.

Moreover, osteoclastogenesis involves differential regulation of the expression of numerous genes $[39,40]$. Particularly, RANKL considerably up-regulates the expression of genes encoding MMP9, Car2, Ctsk, and TRAP [39,40]. Thus, we next investigated whether TZDs affect the expression of these four representative osteoclast genes. As shown in Figure 6C, RANKL activated the expression of MMP9, Ctsk, TRAP and Car2 genes in BMMs at 24, 48 or 96 hours (lanes 2, 7 and 12). Rosiglitazone or pioglitazone markedly suppressed RANKL-induced expression of MMP9, Ctsk, TRAP and Car2 genes in BMMs at 24 (lanes 4-5), 48 (lanes 9-10), and 96 hours (lanes 14-15). The results indicated that TZDS significantly suppress RANKL-mediated expression of 


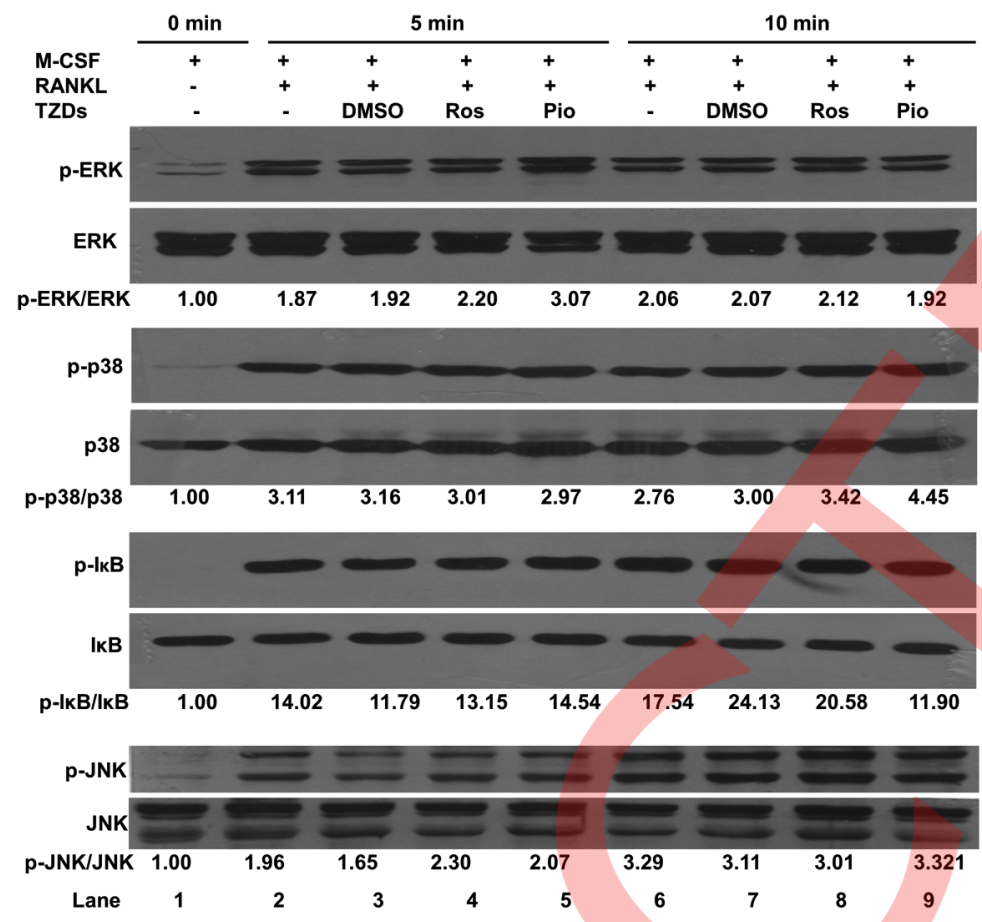

Figure 5. Rosiglitazone and pioglitazone exert no effects on RANKL-induced activation of the NF-KB, JNK, ERK and p38 pathways in BMMs. BMMs were cultured with M-CSF (44 ng/ml) to $20-30 \%$ confluence on $60-\mathrm{mm}$ tissue culture dishes, and then treated with M-CSF (44 $\mathrm{ng} / \mathrm{ml}$ ) alone, M-CSF $(44 \mathrm{ng} / \mathrm{ml})$ and RANKL $(100 \mathrm{ng} / \mathrm{ml})$, M-CSF $(44 \mathrm{ng} / \mathrm{ml})$ and RANKL (100 ng/ml) plus vehicle (DMSO), $40 \mu \mathrm{M}$ rosiglitazone (Ros) or pioglitazone (Pio) for 5 or 10 minutes (min). Activation of ERK, p38, NF- $\mathrm{kB}$ and JNK pathways was assessed as the level of the phosphorylated form of ERK, p38, IKB and JNK using Western analysis with antibodies against phospho-ERK, phospho-p38, phospho-IKB, and phospho-JNK, respectively. The blots were stripped and re-probed with antibodies against ERK, p38 and IKB for the expression levels of total proteins. Separate blots were probed with the antibody against JNK for the expression levels of the total JNK. Ratios of the phosphorylated form to the total protein for these signaling proteins were obtained by dividing the densitometric reading of the phosphorylated form with that of the total protein, and then the value calculated for 0 min was set as 1.00. All assays were repeated three times. One representative set of experiments is shown. doi:10.1371/journal.pone.0102706.g005

NFATc1, MMP9, Ctsk, TRAP and Car2 genes during osteoclastogenesis.

Given that previous studies demonstrated that TZDs inhibit osteoclastogenesis by decreasing the expression of RANK in bone marrow cells and RAW264.7 cells [29,30], we independently examined the effect of rosiglitazone or pioglitazone on RANK expression in BMMs. Towards this end, we treated BMMs with $40 \mu \mathrm{M}$ rosiglitazone or pioglitazone for 1 or 2 days for Western blot analysis of RANK expression. In contrast, our data indicate that these two TZDs do not significantly affect the expression of RANK (lane 2 vs. lanes 3 and 4; also lane 6 vs. lanes 7 and 8, Figure 6D). Therefore, we conclude that TZDs directly inhibit RANKL-induced expression of NFATc1, c-Fos and osteoclast genes, not indirectly through the suppression of RANK expression.

The inhibitory effect of TZDs on osteoclastogenesis is significantly reduced in RANKL-pretreated BMMs

We and others previously established that RANKL-mediated osteoclast lineage commitment dictates the effects of various factor including LPS, IFN- $\gamma$, IL- 1 and TNF- $\alpha$ on osteoclastogenesis [4249]. To determine whether RANKL-mediated osteoclast lineage commitment also modulates the action of TZDs in osteoclastogenesis, rosiglitazone or pioglitazone was added at the beginning of the osteoclastogenic assays, or 24 and 48 hours after the start of the assays. The data revealed that BMMs pretreated with RANKL for as short as 24 hours formed much more osteoclasts compared to BMMs without RANKL pretreatment in the presence of rosiglitazone (Figures 7A-7B) or pioglitazone (Figures 7C-7D). Notably, rosiglitazone or pioglitazone completely abolished osteoclastogenesis from fresh BMMs at the concentration of $40 \mu \mathrm{M}$, replicating our assays above (Figure 3). However, numerous osteoclasts formed from RANKL-pretreated BMMs in the presence of these TZDs at $40 \mu \mathrm{M}$. These findings indicate that RANKL-mediated osteoclast lineage commitment decreases the effects of TZDs on osteoclastogenesis.

To further substantiate this notion, we carried out additional assays to investigate whether RANKL-mediated osteoclast lineage commitment affects the role of TZDs in osteoclastogenesis on bone slices since bone surfaces represent the physiological substratum for osteoclastogenesis and assays with bone slices are more physiologically relevant. Similar to our assays above in Figure 4, no bone resorption pits were found on bone slices with fresh BMMs cultured in the presence of $40 \mu \mathrm{M}$ rosiglitazone (top row, Figure $8 \mathrm{~A}$ ) or $40 \mu \mathrm{M}$ pioglitazone (top row, Figure $8 \mathrm{C}$ ). But, bone slices with RANKL-pretreated BMMs in the presence of $40 \mu \mathrm{M}$ rosiglitazone (middle and bottom rows, Figure $8 \mathrm{~A}$ ) or $40 \mu \mathrm{M}$ pioglitazone (middle and bottom rows, Figure $8 \mathrm{C}$ ) exhibited numerous resorption pits. Quantification of the bone resorption assays indicates that RANKL pretreatment significantly reduced TZD-mediated inhibitory effects on osteoclastogenesis on bone slices (Figures 8B and 8D). These findings further demonstrate that RANKL-mediated osteoclast lineage commitment diminishes the inhibitory effects of TZDs on osteoclastogenesis. 
A
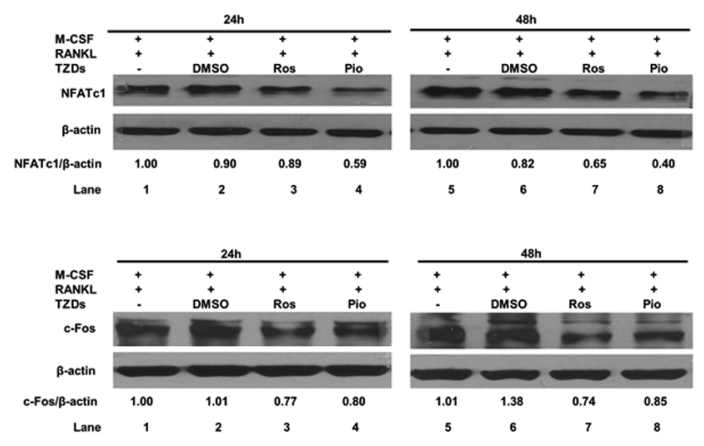

C
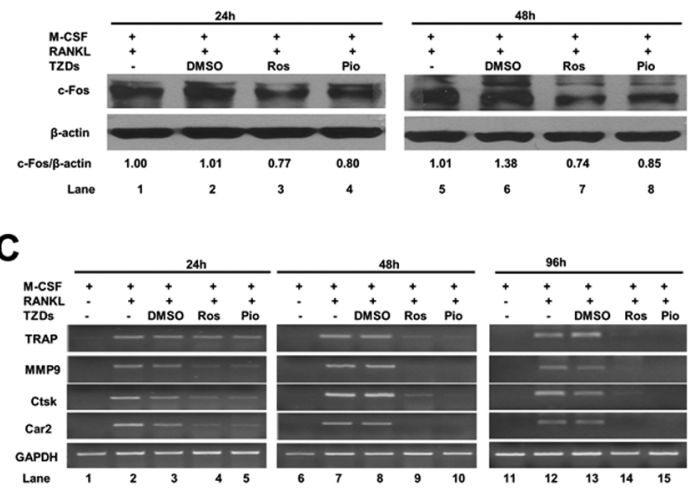

B

D

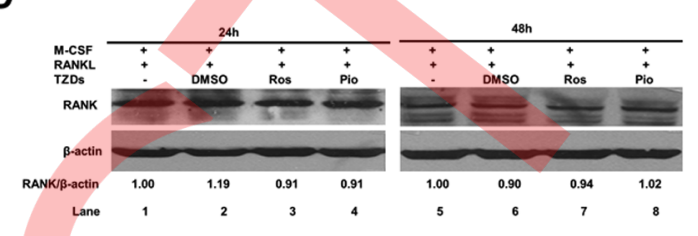

Figure 6. Rosiglitazone and pioglitazone repress RANKL-induced expression of NFATc1, c-Fos, TRAP, MMP9, Ctsk and Car2 genes but exert no effects on RANK expression. (A) BMMs were treated with M-CSF (44 ng/ml) and RANKL (100 ng/ml), M-CSF (44 ng/ml) and RANKL $(100 \mathrm{ng} / \mathrm{ml})$ plus DMSO or $40 \mu \mathrm{M}$ rosiglitazone (Ros) or pioglitazone (Pio) for 24 hours (h), $48 \mathrm{~h}$ prior to lysis for Western blot assays with antibodies against NFATc1 (top panel) or c-Fos (bottom panel). The blots were stripped and then re-probed with $\beta$-actin antibody for loading control. Ratios of NFATc1/c-Fos to $\beta$-actin were obtained by dividing the densitometric reading of NFATc1/c-Fos with that of $\beta$-actin, and then the value calculated for the assay treated with M-CSF $(44 \mathrm{ng} / \mathrm{ml})$ and RANKL $(100 \mathrm{ng} / \mathrm{ml})$ was set as 1.00 . The assays were repeated three times. One representative set of experiments is shown. (B) BMMs were treated with M-CSF (44 ng/ml) and RANKL (100 ng/ml), M-CSF (44 ng/ml) and RANKL (100 ng/ml) plus DMSO or $40 \mu \mathrm{M}$ Ros or Pio for $48 \mathrm{~h}$, and then cytoplasmic and nuclear extracts were prepared from the cells for Western analysis of NFATc1 expression. Lamin A/C was used to determine the quality of the cytoplasmic and nuclear extracts and also used as loading control for nuclear extract samples. $\beta-$ actin was used as loading control for cytoplasmic extract samples. Densitometric values of cytoplasmic NFATc1 were normalized by $\beta$-actin densitometric values, whereas densitometric values of nuclear NFATc1 were normalized by those of lamin A/C. Ratios of nuclear NFATc1 to cytoplasmic NFATc1 were obtained by dividing normalized densitometric values of nuclear NFATc1 with normalized densitometric values of cytoplasmic NFATc1. The assays were repeated two times and one set of data is shown. (C) BMMs were treated with M-CSF (44 ng/ml), M-CSF (44 ng/ $\mathrm{ml})$ and RANKL (100 ng/ml), M-CSF (44 ng/ml) and RANKL (100 ng/ml) plus DMSO, or $40 \mu \mathrm{M}$ Ros/Pio for $24 \mathrm{~h}, 48 \mathrm{~h}$ and $96 \mathrm{~h}$ prior to isolation of total RNAs for semi-quantitative RT-PCR analysis. The assays were repeated three times. One representative set of experiments is shown. (D) BMMs were treated with M-CSF (44 ng/ml) and RANKL (100 ng/ml), M-CSF (44 ng/ml) and RANKL (100 ng/ml) plus DMSO or $40 \mu \mathrm{M}$ Ros/Pio for $24 \mathrm{~h}, 48 \mathrm{~h}$ prior to lysis for Western blot assays with an antibody against RANK. The blots were stripped and then re-probed with $\beta$-actin antibody for loading control. Ratios of RANK to $\beta$-actin were obtained by dividing the densitometric reading of RANK with that of $\beta$-actin, and then the value calculated for the assay treated with M-CSF $(44 \mathrm{ng} / \mathrm{ml})$ and RANKL $(100 \mathrm{ng} / \mathrm{ml})$ was set as 1.00 . All assays were repeated three times. One representative set of experiments is shown.

doi:10.1371/journal.pone.0102706.g006

The inhibitory effect of TZDs on the expression of NFATc1, c-Fos and osteoclast genes is significantly impaired in RANKL-pretreated BMMs

To delineate the molecular mechanism by which RANKLmediated osteoclast lineage commitment modulates the action of TZDs in osteoclastogenesis, we examined the effect of TZDs on the expression of NFATcl and c-Fos in RANKL-pretreated BMMs. Similar to our above assays in Figure 6A, rosiglitazone and pioglitazone dramatically suppressed RANKL-induced expression of NFATc1 (top panel, lane 2 vs. lanes 3 and 4, Figure 9A) and cFos (bottom panel, lane 2 vs. lanes 3 and 4, Figure 9A) in fresh BMMs, the inhibitory effect of these two TZDs on NFATcl (top panel, lane 6 vs. lanes 7 and 8, Figure 9A) and c-Fos (bottom panel, lane 6 vs. lanes 7 and 8, Figure 9A) was almost abrogated when BMMs were pretreated with RANKL. Finally, we also assessed the effects of TZDs on RANKL-mediated expression of the four osteoclast genes in RANKL-pretreated BMMs. Similarly, rosiglitazone or pioglitazone significantly inhibited RANKLinduced expression of MMP9, Ctsk, TRAP and Car2 genes in fresh BMMs (left panel, lane 2 vs. lane 3 and 4, Figure 9B). In contrast, once BMMs were treated with RANKL, the capacity of these two TZDs to suppress RANKL-induced expression of these osteoclast genes was considerably reduced (right panel, lane 6 vs. lanes 7 and 8, Figure 9B). Taken together, these results indicate that RANKL pretreatment reduces the inhibitory effect of TZDs on osteoclastogenesis in part by rendering NFATcl, c-Fos and osteoclast genes refractory to the action of TZDs.

\section{Discussion}

Effects of TZDs on osteoclastogenesis were initially investigated almost 15 years ago by Okazaki and coworkers who carried out their study using mouse whole bone marrow cultures and demonstrated that rosiglitazone, pioglitazone and troglitazone all inhibited osteoclastogenesis in the whole bone marrow culture system [24]. Although whole bone marrow cultures contain different cell types including bone marrow stromal cells, TZDs are likely to exert inhibitory effects on osteoclastogenesis directly by targeting osteoclast precursors rather than indirectly by modulating the production of RANKL and/or OPG by bone marrow stromal cells since these investigators showed that troglitazone did not affect the expression of RANKL or OPG in bone marrow stromal cells [24]. Subsequently, two groups demonstrated that rosiglitazone suppressed RANKL-induced osteoclast formation 

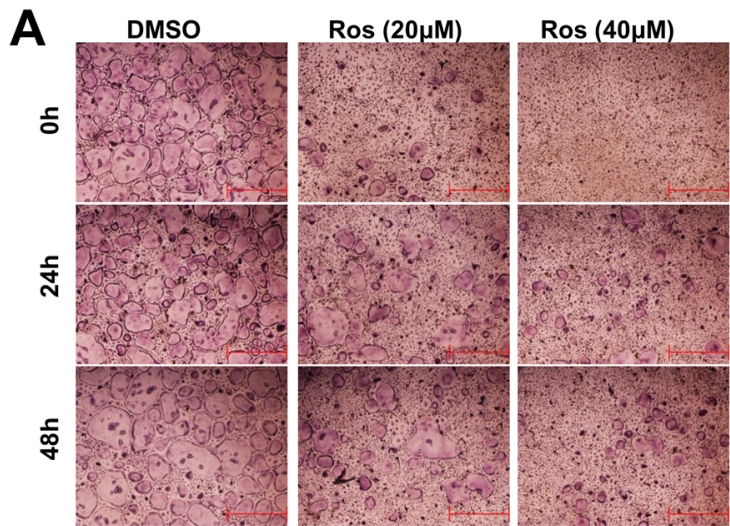

B
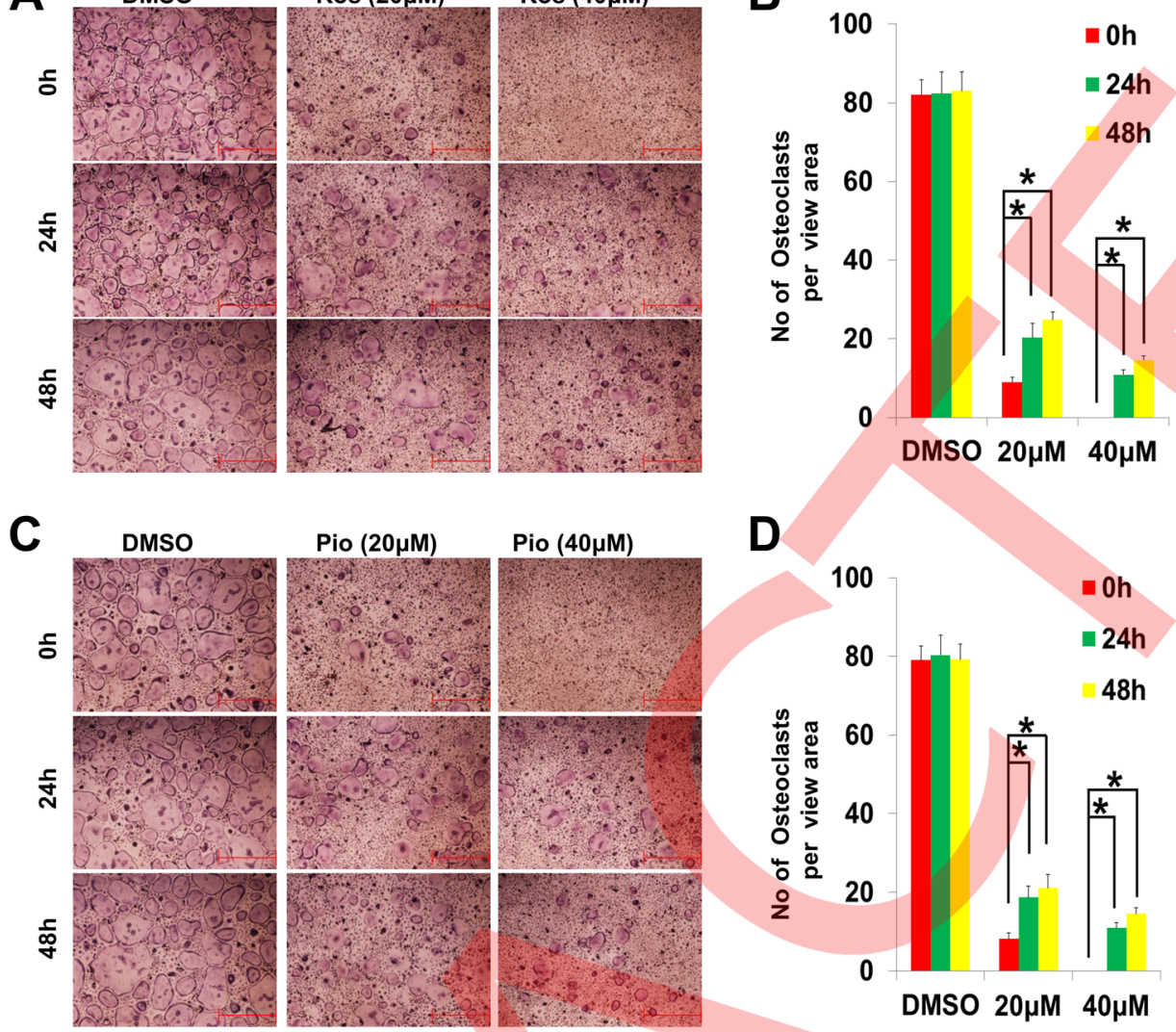

Figure 7. The inhibitory effects of rosiglitazone and pioglitazone on osteoclastogenesis are significantly decreased in RANKLpretreated BMMs. (A) BMMs were cultured with M-CSF (220 ng/ml) until 30-40\% confluence. Then, BMMs were treated with M-CSF (44 $\mathrm{ng} / \mathrm{ml})$ and RNAKL $(100 \mathrm{ng} / \mathrm{ml}$ ) for 4 days to stimulate osteoclastogenesis. Vehicle (DMSO), $20 \mu \mathrm{M}$ or $40 \mu \mathrm{M}$ of rosiglitazone (Ros) was added at the beginning of the assays ( 0 hour $-0 \mathrm{~h}$ ) or $24 \mathrm{~h}$ and $48 \mathrm{~h}$ after the start of the assays. The cultures were stained for TRAP activity. All assays were performed in triplicate and repeated 3 times and one representative view from each condition is shown. (B) Quantification of the osteoclastogenesis assays in A. The number of multinucleated TRAP-positive cells ( $>3$ nuclei) per representative view area at $40 \times$ magnification was obtained. Bars show averages of three replicates \pm S.D. ${ }^{*} P<0.05$. (C) The same set of assays in A was performed with pioglitazone (Pio). (D) The osteoclastogenesis assays in $C$ were quantified as described in $B .{ }^{*} P<0.05$.

doi:10.1371/journal.pone.0102706.g007

from primary bone marrow macrophages [30] and MOCP-5 cells [27], a mouse osteoclast precursor cell line [53]. Pioglitazone was shown to inhibit RANKL-induced osteoclastogenesis from mouse macrophage-like cell line RAW264.7 cells [29]. Moreover, it was also demonstrated that rosiglitazone and pioglitazone block TNF$\alpha$-induced osteoclast differentiation from RAW264.7 cells [28]. These findings indicate that TZDs exert inhibitory effects on murine osteoclastogenesis and they do so directly by targeting osteoclast precursors. Finally, two studies demonstrated that ciglitazone inhibits RANKL- and TNF- $\alpha$-induced differentiation of human peripheral monocytes into osteoclasts [25,26]. Collectively, these seven independent investigations provide several lines of evidence supporting a notion that TZDs exert inhibitory effects on osteoclastogenesis.

Intriguingly, in 2007 Wan and coworkers demonstrated that rosiglitazone enhances RANKL-induced osteoclastogenesis in an PPAR $\gamma$-dependent manner, proposing a concept that TZDs induce bone loss in patients with T2DM in part by promoting osteoclastogenesis [31]. Furthermore, a recent study has also shown that rosiglitazone exerts a stimulatory effect on osteoclastogenesis in vitro [32]. The stimulatory role of TZDs in osteoclastogenesis has been highlighted in several recent review papers $[54,55]$, further promoting the view that TZDs induce bone loss in T2DM patients in part by enhancing osteoclast formation.

Several factors prompted us to carry out this study to further address the role of TZDs in osteoclastogenesis. First, the role of TZDs in osteoclastogenesis remains highly contentious and this controversy raises a concern about the notion that TZDs induce bone loss in T2DM patients in part by enhancing osteoclastogenesis $[54,55]$. We feel that a precise understanding of the role of TZDs in osteoclast formation is a prerequisite for further delineating the mechanism by which TZDs increase fracture risks; Secondly, a better understanding of the role of TZDs in osteoclastogenesis can assist in elucidating the precise role of PPAR $\gamma$ in osteoclastogenesis, which will facilitate future efforts to develop better PPAR $\gamma$ agonists; Thirdly, this study was also promoted by our recent studies revealing that RANKL-mediated osteoclast lineage commitment dictates the effect of various factors such as LPS, IFN- $\gamma$, IL- 1 and TNF- $\alpha$ on osteoclastogenesis [4249]. For example, early investigations on the role of LPS in osteoclastogenesis also generated conflicting results; while the majority of studies showed that LPS stimulates osteoclast formation, a few demonstrated that LPS exerts an inhibitory effect on the process. We later found that LPS inhibits osteoclast formation from normal BMMs but enhances osteoclastogenesis 
A

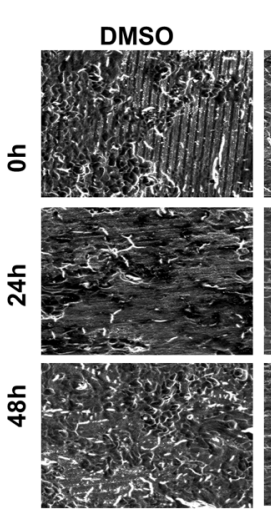

\section{C}

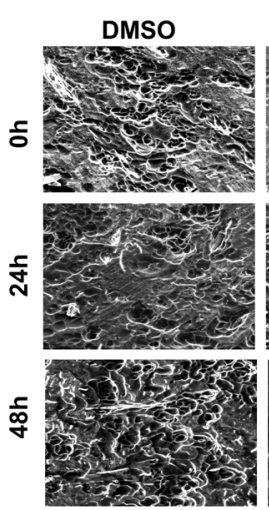

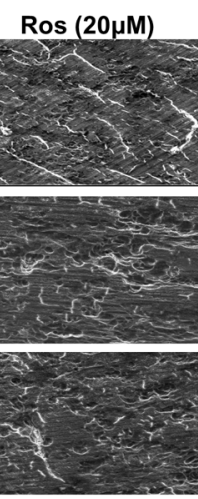

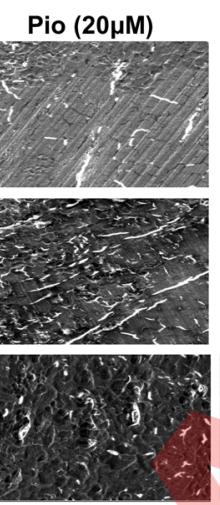

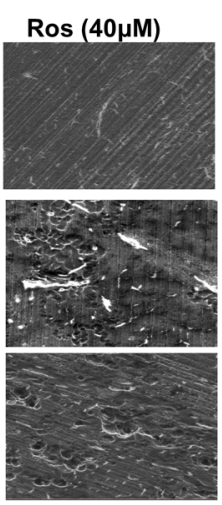

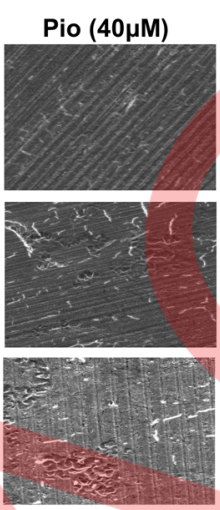

the formation

B

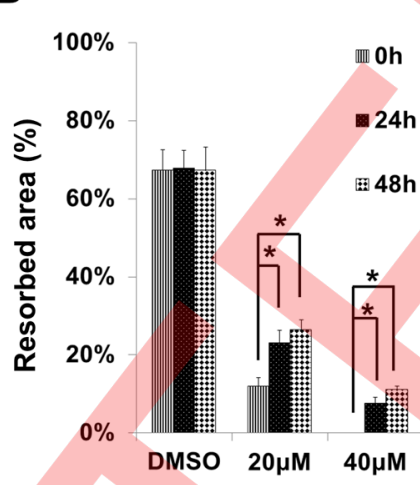

Figure 8. The inhibitory effects of rosiglitazone and pioglitazone on the formation of functional osteoclasts on bone slices are significantly decreased in RANKL-pretreated BMMs. (A) BMMs were seeded on bone slices and treated with M-CSF (44 ng/ml) and RNAKL $(100 \mathrm{ng} / \mathrm{ml}$ ) for 10 days. Vehicle (DMSO), $20 \mu \mathrm{M}$ or $40 \mu \mathrm{M}$ of rosiglitazone (Ros) was added at the beginning of the assays $(0 \mathrm{hour}-0 \mathrm{~h})$, or $24 \mathrm{~h}$ and $48 \mathrm{~h}$ after the start of the assays. Bone resorption pits were visualized by SEM and one representative view from each condition is shown. All assays were performed in duplicates and were repeated 2 times. (B) Quantification of bone resorption assays for rosiglitazone in A. 5 view areas on each bone slice were randomly chosen. Bars show mean percentage of resorbed bone area $\pm S . D$. ${ }^{*} P<0.05$. (C) The same set of assays in $A$ was performed with pioglitazone (Pio). (D) The bone resorption assays in $C$ were quantified as described in $B .{ }^{*} P<0.05$.

doi:10.1371/journal.pone.0102706.g008

when BMMs are pretreated with RANKL [42]; this led to a realization that the conflicting data on the role of LPS in osteoclastogenesis might result from cells with different states of lineage commitment used by different groups. So, our initial hypothesis was that RANKL-mediated osteoclast lineage commitment also modulates the effect of TZDs on osteoclastogenesis; specifically, TZDs inhibit osteoclastogenesis from normal BMMs but enhances osteoclastogenesis from RANKL-pretreated BMMs. Finally, a need for further studies to address the controversy is also raised by other investigators [18].

In light of the controversy, we carried out a series of in vitro osteoclastogenesis assays in this study to independently and thoroughly investigate the effect of TZDs on osteoclastogenesis using primary BMMs (namely, authentic osteoclast precursors) and two TZDs (rosiglitazone and pioglitazone). More importantly, we also validated key osteoclastogenesis data obtained from tissue culture plates by performing bone resorption assays to assess the formation of functional osteoclasts on bone slices, which represent the physiological substratum for osteoclastogenesis. We found that both rosiglitazone and pioglitazone dose-dependently inhibit osteoclastogenesis in vitro from normal BMMs (namely, primary BMMs which were freshly isolated and not subjected to treatments with RANKL or any other factors), replicating the findings from these seven previous studies [24-30]. To test our hypothesis that TZDs inhibit osteoclastogenesis from normal BMMs but enhances osteoclastogenesis from RANKL-pretreated BMMs, we further determined whether rosiglitazone and pioglitazone can promote osteoclastogenesis with low levels of RANKL or from RANKLpretreated BMMs. Unexpectedly, we found that neither rosiglitazone nor pioglitazone was able to mediate osteoclastogenesis in the presence of low levels of RANKL or from RANKL-pretreated BMMs. Nonetheless, we found that the inhibitory effect of both rosiglitazone and pioglitazone is significantly reduced in RANKLpretreated BMMs compared to normal BMMs. Thus, our data indicate that rosiglitazone and pioglitazone are unable to enhance osteoclastogenesis under any circumstances and this finding is inconsistent with those from two previous studies [31,32].

Obviously, the inhibitory effect of TZDs on osteoclastogenesis is counterintuitive given that clinical data indicate that TZD treatments cause bone loss and increase fracture risks in T2DM patients. So, how can our findings explain the clinical observation? To address this issue, we would like to highlight following important observations: a) TZDs inhibit osteoblast differentiation and bone formation [19-21]; b) TZDs induce bone loss in ovariectomized rats but not in intact rats [56]; c) TZD-induced bone loss is more pronounced in postmenopausal women [57], who have increased levels of RANKL expression on bone marrow cells compared to premenopausal counterparts [58]. Based on these observations and our current findings, we propose the following hypothesis to explain how TZDs induce bone loss and 
A
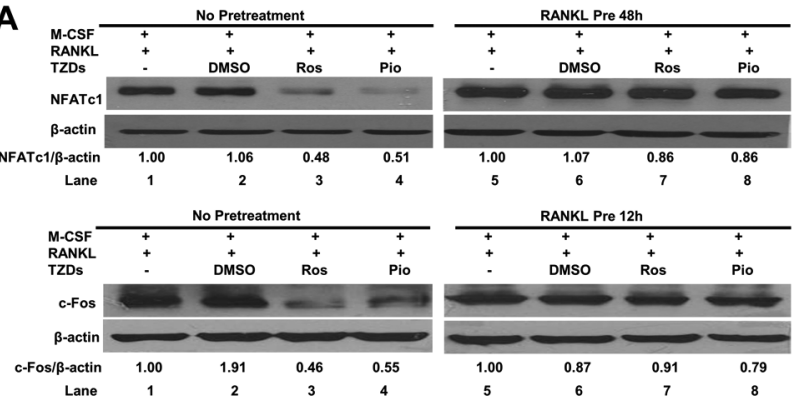

B
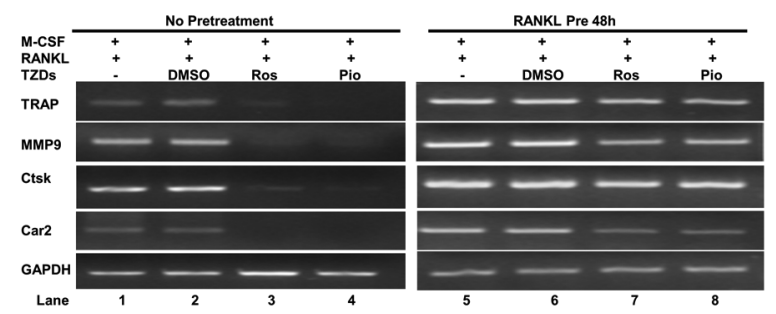

Figure 9. The inhibitory effects of rosiglitazone and pioglitazone on the expression of NFATc1, c-Fos, TRAP, MMP9, Ctsk and Car2 genes are significantly impaired in RANKL-pretreated BMMs. (A) Fresh BMMs (No Pretreatment) or BMMs pretreated with $\mathrm{M}$ CSF (44 $\mathrm{ng} / \mathrm{ml})$ and RANKL (100 $\mathrm{ng} / \mathrm{ml})$ for $48 \mathrm{~h}$ (RANKL Pre $48 \mathrm{~h}$ ) were treated with M-CSF $(44 \mathrm{ng} / \mathrm{ml})$ and RANKL $(100 \mathrm{ng} / \mathrm{ml})$, M-CSF (44 ng/ $\mathrm{ml}$ ) and RANKL (100 ng/ml) plus DMSO, $40 \mu \mathrm{M}$ rosiglitazone (Ros) or pioglitazone (Pio) for 48 hours (h). Cells were then lysed for Western blot analysis of NFATc1 expression. For c-Fos assays, BMMs were pretreated with RANKL for $12 \mathrm{~h}$ before culturing with two TZDs for additional $12 \mathrm{~h}$ prior to Western blot analysis. The blots were stripped and then re-probed with $\beta$-actin antibody for loading control. Ratios of NFATc1/c-Fos to $\beta$-actin were obtained by dividing the densitometric reading of NFATC1/c-Fos with that of $\beta$-actin, and then the value calculated for the assay treated with M-CSF $(44 \mathrm{ng} / \mathrm{ml})$ and RANKL $(100 \mathrm{ng} / \mathrm{ml})$ was set as 1.00 . The assays were repeated 3 times and the same results were obtained. One typical set of assays is shown. (B) The same set of cultures in A for NFATC1 was repeated for semi-quantitative RT-PCR analysis of the expression of TRAP, MMP9, Ctsk and Car2 genes using GAPDH gene as control. The assays were repeated 3 times and the same results were obtained. One set of assays is shown. doi:10.1371/journal.pone.0102706.g009

increase fracture risks in postmenopausal T2DM patients: in premenopausal T2DM patients, TZDs inhibit both osteoblast differentiation (bone formation) and osteoclast differentiation (bone resorption) probably to similar extents, thus resulting in no significant net change in bone mass. However, the onset of menopause leads to increases in RANKL levels, which augment the commitment of BMMs into the osteoclast lineage. As a consequence, the inhibitory effect of TZDs on osteoclast differentiation (bone resorption) is reduced based on our current finding while the extent of TZDs-mediated inhibition on osteoblast differentiation (bone formation) remains the same; this therefore leads to bone loss and increases in fracture risks in postmenopausal T2DM patients. Future studies should be carried out to test this hypothesis using ovariectomized mouse and/or rat models.

We extended our study to investigate the molecular mechanism by which TZDs suppress osteoclastogenesis. Our data indicate that rosiglitazone and pioglitazone inhibit osteoclastogenesis not through impairing RANKL-induced activation of the NF- $\mathrm{B}$, JNK, p38 and ERK pathways in BMMs. However, both rosiglitazone and pioglitazone inhibit RANKL-induced expression of NFATcl and c-Fos, two key transcriptional regulators of osteoclastogenesis $[37,38]$, and four osteoclast genes (TRAP, Ctsk,
MMP9 and Car2). This observation is consistent with previous studies showing that rosiglitazone inhibits osteoclastogenesis from MOCP-5 osteoclast precursor cell line by down-regulating TRAP expression [27] and that rosiglitazone and pioglitazone block TNF- $\alpha$-induced osteoclastogenesis by suppressing NFATcl expression [28]. Interestingly, two groups demonstrated that RANK expression was down-regulated by rosiglitazone in BMMs [30] and by pioglitazone in RAW264.7 cells [29], suggesting that these TZDs may inhibit osteoclastogenesis by down-regulating RANK expression. It is noted that Wan et al previously showed that rosiglitazone not only enhances osteoclastogenesis but also mechanistically does so by up-regulating the expression of NFATcl and the four osteoclast genes [31]. Their findings completely conflicts with ours in this study in that rosiglitazone and pioglitazone inhibits osteoclastogenesis in part by downregulating the expression of NFATc1, c-Fos and the four osteoclast genes, and even in RANKL-pretreated BMMs rosiglitazone and pioglitazone were unable to up-regulate the expression of NFATcl and the four osteoclast genes.

Our study has also raised several crucial issues which need to be further addressed to gain a complete understanding of the role and mechanisms of TZDs in osteoclast biology. The first question is whether TZDs mediate the inhibitory effects on osteoclastogenesis through PPAR $\gamma$. Although TZDs were established as agonists of PPAR $\gamma$, these synthetic compounds may also bind to other intracellular molecules. So, future studies are needed to address whether TZDs inhibit osteoclastogenesis in PPAR $\gamma$-dependent or independent manner. Secondly, if TZDs inhibit osteoclastogenesis in PPAR $\gamma$-dependent fashion, a question arises regarding the molecular mechanism by which TZDs suppress osteoclastogenesis. Elucidation of the molecular mechanism underlying the inhibitory role of TZDs in osteoclastogenesis may guide the future development of new and better PPAR $\gamma$ agonists for therapy. Thirdly, it is noted that Wan et al investigated the role of PPAR $\gamma$ in osteoclastogenesis in vivo using a mouse model in which PPAR $\gamma$ is deleted in endothelial cells and multiple hematopoietic lineages [31]. If future in vitro studies demonstrate that TZDs inhibit osteoclastogenesis in PPAR $\gamma$-dependent fashion, new animal models in which PPAR $\gamma$ is selectively deleted in osteoclast precursors (namely, hematopoietic cells of the monocyte/macrophage lineage) should be developed to better address the role of PPAR $\gamma$ in osteoclastogenesis in vivo.

In summary, the findings from this study combined with those from seven previous studies collectively support the notion that TZDs inhibit osteoclastogenesis. Moreover, our study also reveals new insights into the molecular mechanism by which TZDs inhibit osteoclastogenesis. These findings are significant since the precise understanding of the role of TZDs on osteoclastogenesis in vitro is required for designing proper animal model studies to delineate the mechanisms by which TZDs induce bone loss in vivo. Furthermore, our findings in this study highlight the importance of further investigation of the role of PPAR $\gamma$ in osteoclastogenesis in vitro and in vivo. These future studies will not only provide a better understanding of the role and mechanisms of TZDs and PPAR $\gamma$ in osteoclast biology but, more importantly, also facilitate development of better PPAR $\gamma$ agonists with no or fewer side effects for T2DM patients.

\section{Acknowledgments}

We thank Joel Jules, Erin M. McCoy and Huaping Chen in the Department of Pathology, and Yan Li in the Department of Nutritional Sciences at UAB for their expert technical assistance and Robin D. Foley in the Department of Materials Science \& Engineering at UAB for her assistance in performing SEM and data analysis. Disclaimer: The content is 
solely the responsibility of the authors and does not necessarily represent the official views of the National Institutes of Health.

\section{References}

1. Shaw JE, Sicree RA, Zimmet PZ (2010) Global estimates of the prevalence of diabetes for 2010 and 2030. Diabetes Res Clin Pract 87: 4-14.

2. Zhang P, Zhang X, Brown J, Vistisen D, Sicree R, et al. (2010) Global healthcare expenditure on diabetes for 2010 and 2030. Diabetes Res Clin Pract 87: 293-301

3. Krishnaswami A, Ravi-Kumar S, Lewis JM (2010) Thiazolidinediones: a 2010 perspective. Perm J 14: 64-72.

4. Krentz AJ, Bailey CJ (2005) Oral antidiabetic agents: current role in type 2 diabetes mellitus. Drugs 65: 385-411.

5. Reginato MJ, Lazar MA (1999) Mechanisms by which Thiazolidinediones Enhance Insulin Action. Trends Endocrinol Metab 10: 9-13.

6. Tontonoz P, Spiegelman BM (2008) Fat and beyond: the diverse biology of PPARgamma. Annual review of biochemistry 77: 289-312.

7. Kim HI, Ahn YH (2004) Role of peroxisome proliferator-activated receptorgamma in the glucose-sensing apparatus of liver and beta-cells. Diabetes 53 Suppl 1: S60-65.

8. Barroso I, Gurnell M, Crowley VE, Agostini M, Schwabe JW, et al. (1999) Dominant negative mutations in human PPARgamma associated with severe insulin resistance, diabetes mellitus and hypertension. Nature 402: 880-883

9. Tontonoz P, Hu E, Spiegelman BM (1994) Stimulation of adipogenesis in fibroblasts by PPAR gamma 2, a lipid-activated transcription factor. Cell 79: $1147-1156$

10. Tontonoz P, Nagy L, Alvarez JG, Thomazy VA, Evans RM (1998) PPARgamma promotes monocyte/macrophage differentiation and uptake of oxidized LDL. Cell 93: 241-252.

11. Chawla A, Boisvert WA, Lee CH, Laffitte BA, Barak Y, et al. (2001) A PPAR gamma-LXR-ABCAl pathway in macrophages is involved in cholesterol efflux and atherogenesis. Mol Cell 7: 161-171.

12. Jiang C, Ting AT, Seed B (1998) PPAR-gamma agonists inhibit production of monocyte inflammatory cytokines. Nature 391: 82-86.

13. Ricote M, Li AC, Willson TM, Kelly CJ, Glass CK (1998) The peroxisome proliferator-activated receptor-gamma is a negative regulator of macrophage activation. Nature 391: 79-82.

14. Lehrke M, Lazar MA (2005) The many faces of PPARgamma. Cell 123: 993 999.

15. Moseley KF (2012) Type 2 diabetes and bone fractures. Curr Opin Endocrinol Diabetes Obes 19: 128-135.

16. Betteridge DJ (2011) Thiazolidinediones and fracture risk in patients with Type 2 diabetes. Diabet Med 28: 759-771.

17. Bazelier MT, de Vries F, Vestergaard P, Herings RM, Gallagher AM, et al. (2013) Risk of fracture with thiazolidinediones: an individual patient data metaanalysis. Front Endocrinol (Lausanne) 4: 11.

18. Mabilleau G, Chappard D, Basle MF (2011) Cellular and molecular effects of thiazolidinediones on bone cells: a review. Int J Biochem Mol Biol 2: 240-246.

19. Lecka-Czernik B, Moerman EJ, Grant DF, Lehmann JM, Manolagas SC, et al. (2002) Divergent effects of selective peroxisome proliferator-activated receptorgamma 2 ligands on adipocyte versus osteoblast differentiation. Endocrinology 143: 2376-2384

20. Ali AA, Weinstein RS, Stewart SA, Parfitt AM, Manolagas SC, et al. (2005) Rosiglitazone causes bone loss in mice by suppressing osteoblast differentiation and bone formation. Endocrinology 146: 1226-1235.

21. Rzonca SO, Suva LJ, Gaddy D, Montague DC, Lecka-Czernik B (2004) Bone is a target for the antidiabetic compound rosiglitazone. Endocrinology 145: 401406

22. Soroceanu MA, Miao D, Bai XY, Su H, Goltzman D, et al. (2004) Rosiglitazone impacts negatively on bone by promoting osteoblast/osteocyte apoptosis. J Endocrinol 183: 203-216.

23. Mabilleau G, Mieczkowska A, Edmonds ME (2010) Thiazolidinediones induce osteocyte apoptosis and increase sclerostin expression. Diabet Med 27: 925-932.

24. Okazaki R, Toriumi M, Fukumoto S, Miyamoto M, Fujita T, et al. (1999) Thiazolidinediones inhibit osteoclast-like cell formation and bone resorption in vitro. Endocrinology 140: 5060-5065.

25. Chan BY, Gartland A, Wilson PJ, Buckley KA, Dillon JP, et al. (2007) PPAR agonists modulate human osteoclast formation and activity in vitro. Bone 40: 149-159.

26. Hounoki H, Sugivama E, Mohamed SG, Shinoda K, Taki H, et al. (2008) Activation of peroxisome proliferator-activated receptor gamma inhibits TNFalpha-mediated osteoclast differentiation in human peripheral monocytes in part via suppression of monocyte chemoattractant protein-1 expression. Bone 42: 765-774.

27. Hassumi MY, Silva-Filho VJ, Campos-Junior JC, Vieira SM, Cunha FQ et al. (2009) PPAR-gamma agonist rosiglitazone prevents inflammatory periodontal bone loss by inhibiting osteoclastogenesis. International immunopharmacology 9: $1150-1158$.

\section{Author Contributions}

Conceived and designed the experiments: XF YW AHW. Performed the experiments: DZ ZS HH PQ. Analyzed the data: DZ ZS HH PQ. Wrote the paper: DZ YW AHW XF.

28. Yang CR, Lai CC (2010) Thiazolidinediones inhibit TNF-alpha-mediated osteoclast differentiation of RAW264.7 macrophages and mouse bone marrow cells through downregulation of NFATc1. Shock 33: 662-667.

29. Jiang M, Lu S, Wu L, Liu J, Cheng P, et al. (2010) [Effect of pioglitazone on receptor activator of nuclear factor-kappa B expression in osteoclast from RAW264.7 cells]. Zhonghua Yi Xue Za Zhi 90: 2282-2285.

30. Cho ES, Kim MK, Son YO, Lee KS, Park SM, et al. (2012) The effects of rosiglitazone on osteoblastic differentiation, osteoclast formation and bone resorption. Mol Cells 33: 173-181.

31. Wan Y, Chong LW, Evans RM (2007) PPAR-gamma regulates osteoclastogenesis in mice. Nature medicine 13: 1496-1503.

32. Wu H, Li L, Ma Y, Chen Y, Zhao J, et al. (2013) Regulation of selective PPARgamma modulators in the differentiation of osteoclasts. J Cell Biochem 114: 1969-1977.

33. Teitelbaum SL (2007) Osteoclasts: what do they do and how do they do it? Am J Pathol 170: 427-435

34. Feng X, McDonald JM (2011) Disorders of bone remodeling. Annu Rev Pathol 6: $121-145$.

35. Boyle WJ, Simonet WS, Lacey DL (2003) Osteoclast differentiation and activation. Nature 423: 337-342.

36. Feng X (2005) Regulatory roles and molecular signaling of TNF family members in osteoclasts. Gene 350: 1-13.

37. Takayanagi H, Kim S, Koga T, Nishina H, Isshiki M, et al. (2002) Induction and activation of the transcription factor NFATc1 (NFAT2) integrate RANKL signaling in terminal differentiation of osteoclasts. Dev Cell 3: 889-901.

38. Grigoriadis AE, Wang ZQ, Cecchini MG, Hofstetter W, Felix R, et al. (1994) cFos: a key regulator of osteoclast-macrophage lineage determination and bone remodeling. Science 266: 443-448.

39. Cappellen D, Luong-Nguyen NH, Bongiovanni S, Grenet O, Wanke C, et al. (2002) Transcriptional program of mouse osteoclast differentiation governed by the macrophage colony-stimulating factor and the ligand for the receptor activator of NFkappa B. J Biol Chem 277: 21971-21982.

40. Rho J, Altmann CR, Socci ND, Merkov L, Kim N, et al. (2002) Gene expression profiling of osteoclast differentiation by combined suppression subtractive hybridization (SSH) and cDNA microarray analysis. DNA Cell Biol 21: 541549.

41. Xu D, Wang S, Liu W, Liu J, Feng X (2006) A novel receptor activator of NFkappaB (RANK) cytoplasmic motif plays an essential role in osteoclastogenesis by committing macrophages to the osteoclast lineage. The Journal of biological chemistry 281: 4678-4690.

42. Liu J, Wang S, Zhang P, Said-Al-Naief N, Michalek SM, et al. (2009) Molecular mechanism of the bifunctional role of lipopolysaccharide in osteoclastogenesis. The Journal of biological chemistry 284: 12512-12523.

43. Zou W, Bar-Shavit Z (2002) Dual modulation of osteoclast differentiation by lipopolysaccharide. Journal of bone and mineral research : the official journal of the American Society for Bone and Mineral Research 17: 1211-1218.

44. Huang W, O'Keefe RJ, Schwarz EM (2003) Exposure to receptor-activator of NFkappaB ligand renders pre-osteoclasts resistant to IFN-gamma by inducing terminal differentiation. Arthritis Res Ther 5: R49-59.

45. Cheng J, Liu J, Shi Z, Jules J, Xu D, et al. (2012) Molecular mechanisms of the biphasic effects of interferon-gamma on osteoclastogenesis. J Interferon Cytokine Res 32: 34-45.

46. Lam J, Takeshita S, Barker JE, Kanagawa O, Ross FP, et al. (2000) TNF-alpha induces osteoclastogenesis by direct stimulation of macrophages exposed to permissive levels of RANK ligand. The Journal of clinical investigation 106: 1481-1488.

47. Jules J, Shi Z, Liu J, Xu D, Wang S, et al. (2010) Receptor activator of NF\{kappa\}B (RANK) cytoplasmic IVVY535-538 motif plays an essential role in tumor necrosis factor-\{alpha\} (TNF)-mediated osteoclastogenesis. The Journal of biological chemistry 285: 37427-37435.

48. Jules J, Zhang P, Ashley JW, Wei S, Shi Z, et al. (2012) Molecular basis of requirement of receptor activator of nuclear factor kappaB signaling for interleukin 1-mediated osteoclastogenesis. J Biol Chem 287: 15728-15738.

49. Wei S, Kitaura H, Zhou P, Ross FP, Teitelbaum SL (2005) IL-1 mediates TNFinduced osteoclastogenesis. The Journal of clinical investigation 115: 282-290.

50. McHugh KP, Hodivala-Dilke K, Zheng MH, Namba N, Lam J, et al. (2000) Mice lacking beta3 integrins are osteosclerotic because of dysfunctional osteoclasts. The Journal of clinical investigation 105: 433-440.

51. Takeshita S, Kaji K, Kudo A (2000) Identification and characterization of the new osteoclast progenitor with macrophage phenotypes being able to differentiate into mature osteoclasts. J Bone Miner Res 15: 1477-1488.

52. Jules J, Zhang P, Ashley JW, Wei S, Shi Z, et al. (2012) Molecular basis of requirement of receptor activator of nuclear factor kappaB signaling for interleukin 1-mediated osteoclastogenesis. The Journal of biological chemistry 287: 15728-15738. 
53. Chen W, Li YP (1998) Generation of mouse osteoclastogenic cell lines immortalized with SV40 large T antigen. J Bone Miner Res 13: 1112-1123.

54. Wan Y (2010) PPARgamma in bone homeostasis. Trends Endocrinol Metab 21: $722-728$.

55. Ahmadian M, Suh JM, Hah N, Liddle C, Atkins AR, et al. (2013) PPARgamma signaling and metabolism: the good, the bad and the future. Nat Med 19: 557566

56. Sottile V, Seuwen K, Kneissel M (2004) Enhanced marrow adipogenesis and bone resorption in estrogen-deprived rats treated with the PPARgamma agonist BRL49653 (rosiglitazone). Calcified tissue international 75: 329-337.
57. Grey A, Bolland M, Gamble G, Wattie D, Horne A, et al. (2007) The peroxisome proliferator-activated receptor-gamma agonist rosiglitazone decreases bone formation and bone mineral density in healthy postmenopausal women: a randomized, controlled trial. The Journal of clinical endocrinology and metabolism 92: 1305-1310.

58. Eghbali-Fatourechi G, Khosla S, Sanyal A, Boyle WJ, Lacey DL, et al. (2003) Role of RANK ligand in mediating increased bone resorption in early postmenopausal women. The Journal of clinical investigation 111: 1221-1230.

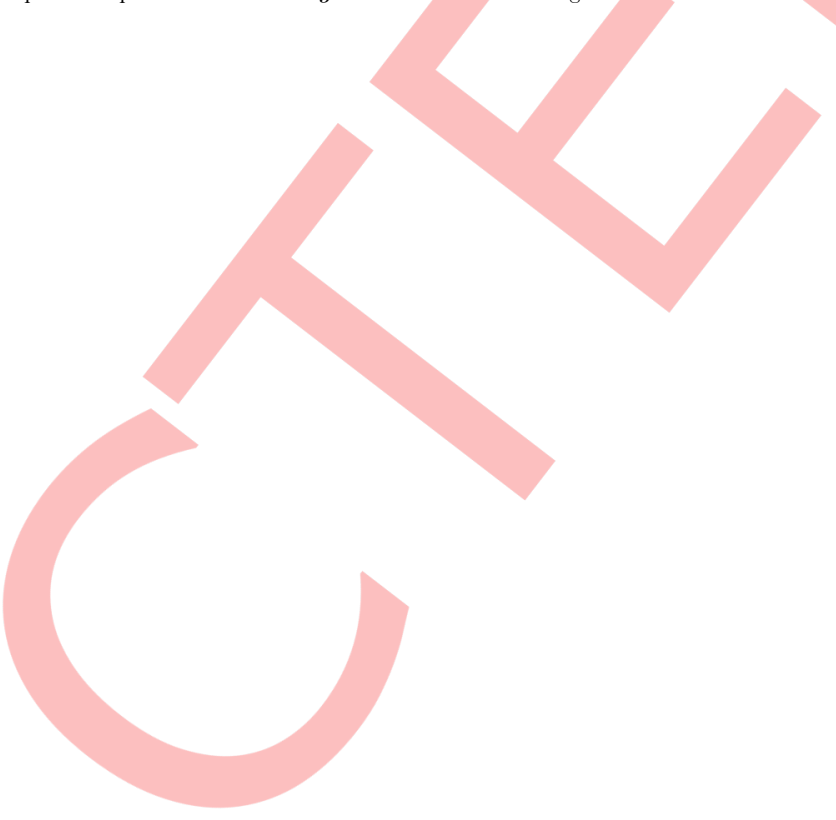

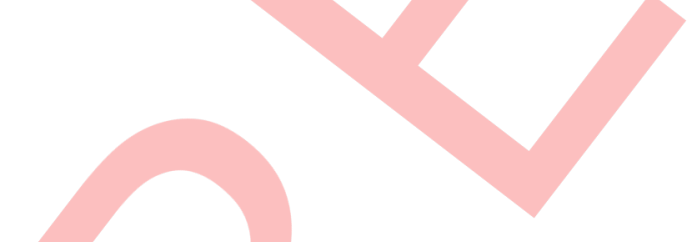

